\title{
Avaliação da atividade antimicrobiana de duas espécies de Rutaceae do Nordeste Brasileiro
}

\author{
Cinara V. da Silva, ${ }^{, 1}$ André L. V. Reis, ${ }^{2}$ Suzana R. Ferrer, ${ }^{2}$ Hygia M. N. Guerreiro, ${ }^{2}$ \\ Tânia F. Barros, ${ }^{2}$ Eudes da S. Velozo ${ }^{l}$
}

\begin{abstract}
${ }^{1}$ Laboratório de Pesquisa em Matéria Médica, Departamento do Medicamento. Faculdade de Farmácia da Universidade Federal da Bahia. Rua Barão de Jeremoabo s/n, Campus Universitário de Ondina, 40170-290 Salvador-BA, Brasil,

${ }^{2}$ Laboratório de Pesquisa em Microbiologia Clínica, Departamento de Análises Clínicas e Toxicológicas, Faculdade de Farmácia da Universidade Federal da Bahia. Rua Barão de Jeremoabo s/n, Campus Universitário de Ondina,

40170-290 Salvador-BA, Brasil.
\end{abstract}

\begin{abstract}
RESUMO: Com o aumento da resistência bacteriana aos antibióticos disponíveis, tornouse imprescindível a busca por novos fármacos ou protótipos. Os metabólitos secundários produzidos por alguns vegetais como cumarinas, alcaloides e terpenoides podem apresentar várias atividades biológicas, dentre elas, atividade antibiótiotica. O objetivo deste trabalho foi avaliar a atividade antimicrobiana in vitro, pelo método de difusão em disco, das diferentes partes de duas espécies pertencentes à família Rutaceae coletadas na Chapada Diamantina, Bahia, Brasil: Spiranthera odoratissima A. St.-Hil. e Zanthoxylum stelligerum Turcz., bem como do alcaloide diidroqueleritrina, isolado do extrato metanólico de Z. stelligerum frente a cepas padrão de microrganismos e isolados clínicos. Os resultados apresentados indicam que o extrato da raiz da espécie Z. stelligerum e o alcaloide extraído desta apresentaram propriedades antimicrobianas contra as cepas Gram positivas e leveduras. A E. coli foi a única cepa Gram negativa que se mostrou sensível ao extrato e ao alcaloide.
\end{abstract}

Unitermos: Spiranthera odoratissima, Zanthoxylum stelligerum, atividade antimicrobiana, diidroqueleritrina, Rutaceae.

\begin{abstract}
Evaluation of the antimicrobial activity of two Rutaceae species from the Brazilian Northeast". With the increase in bacterial resistance to available antibiotics, it became imperative to search for new drugs or prototypes. The secondary metabolites produced by some plants as coumarins, alkaloids and terpenoids have several biological activities, among them, antibiotic activity. The objective of this study was to evaluate the antimicrobial activity in vitro by the disk diffusion method, from different parts of two species belonging to the family Rutaceae, collected in the Chapada Diamantina, Bahia, Brazil: Spiranthera odoratissima A. St Hil. and Zanthoxylum stelligerum Turcz., and the alkaloid dihydrochelerythrine, isolated from the methanolic extract of Z. stelligerum front of standard strains of microorganisms and clinical isolates. The results indicate that the extract from the roots of $Z$. stelligerum and the alkaloid had antimicrobial properties against Gram positive and yeast strains. The $E$. coli was the only Gram negative strain that was sensitive to extract and the alkaloid.
\end{abstract}

Keywords: Zanthoxylum stelligerum, Spiranthera odoratissima, antimicrobial activity, dihydrochelerythrine, Rutaceae.

\section{INTRODUÇÃO}

O surgimento dos antibióticos e quimioterápicos permitiu o controle das doenças infecciosas, mudando a evolução natural destas enfermidades de modo marcante e sendo determinante na cura de milhares de pessoas. No entanto, apenas alguns anos após o início do uso clínico da penicilina, o primeiro antibacteriano comercializado, já havia sido registrado o primeiro caso de resistência bacteriana adquirida (Moreira, 2004).

Atualmente, a resistência bacteriana vem sendo considerada um crescente problema de saúde pública mundial e o maior obstáculo para o sucesso de um tratamento, já que continua a reduzir o número de antibióticos válidos disponíveis (Oliveira et al., 2008; Banghezal et al., 2007).

A resistência bacteriana é um fenômeno relacionado à formação de cepas não sensíveis aos 
antibióticos, que são capazes de se multiplicar mesmo na presença de concentrações de antimicrobianos mais elevadas do que as provenientes de doses terapêuticas convencionais e; ocorre devido à evolução natural dos microrganismos, além do uso desmedido e irracional destes agentes nas práticas médicas, agrárias e veterinárias (Wannmacher, 2004; Hoefler et al., 2006).

Apesar de todos os esforços da comunidade científica, a descoberta de novos antibióticos tem atingido sucesso limitado (Wang et al., 2006). Várias medidas tecnológicas são sugeridas para resolver o problema das bactérias multirresistentes, entre elas, a busca por novos medicamentos ou protótipos oriundos de espécies vegetais (Haida et al., 2007).

Algumas espécies vegetais sintetizam como metabólitos secundários, substâncias de defesa contra predadores: microrganismos patogênicos, insetos e animais herbívoros (Alves, 2001; Dewick, 2002). Estes compostos despertam grande interesse pelas atividades biológicas produzidas em resposta ao meio ambiente e efeitos fisiológicos que exercem nos animais (Alves, 2001; Robbers et al., 1996). São produtos quimicamente variados, sendo de particular interesse os terpenoides, alcaloides e cumarinas, por apresentarem ação antimicrobiana com grande frequência (Haida et al., 2007).

Muitos estudos têm sido realizados com espécies da família Rutaceae, devido à sua distribuição limitada, biogênese razoavelmente definida, além da diversidade de micromoléculas já descritas. Gibbs (1974) afirma que a família é muito bem caracterizada pela variedade de alcaloides e cumarinas que apresenta com potencial atividade microbiológica (Silva, 2006). Encontra-se amplamente distribuída na Chapada Diamantina e região do semi-árido baiano.

Dentre as espécies de Rutaceae encontradas na Chapada Diamantina, duas destacam-se: Zanthoxylum stelligerum Turcz. e Spiranthera odoratissima A. St Hil., empregadas na medicina popular contra distúrbios inflamatórios ou infecções (Oliveira et al., 2002).

$\mathrm{O}$ estudo fitoquímico das raízes de $Z$. stelligerum apresentou como resultado o isolamento de quatro alcaloides: diidroqueleretrina (1), dictamina (2), robustina (3) e esquimianina (4); um triterpenoide: lupeol (5); uma furanocumarina: imperatorina (6); uma lignana sesamina (7) e dois esteroides: estigmasterol (8) e $\beta$-sitosterol (9) (Silva et al., 2002).

O perfil químico de Spiranthera odoratissima (A. St Hil.) apontou a presença de monoterpenos: pineno (10), limoneno (11), mirceno (12); os sesquiterpenos: copaeno (13), germacreno B (14) e D (15), cariofileno (16), espatulenol (17) e óxido de cariofileno (18); as cumarinas: aurapteno (19), ostol (20), brailina (21) e os alcaloides furoquinolínicos: esquimianina (4) e $\gamma$-fagarina (22) (Freitas et al., 2003).<smiles>COc1ccc2c(c1OC)CN(C)c1c-2ccc2cc3c(cc12)OCO3</smiles>

1<smiles>CC(C)=CCOc1c2occc2cc2ccc(=O)oc12</smiles>

6<smiles>[R]c1cc2c(OC)c3ccoc3nc2c([R3])c1[R2]</smiles>

2 - $\mathrm{R}_{1}=\mathrm{R}_{2}=\mathrm{R}_{3}=\mathrm{H}$

$3-\mathrm{R}_{1}=\mathrm{R}_{2}=\mathrm{H} ; \mathrm{R}_{3}=\mathrm{OH}$

$4-\mathrm{R}_{1}=\mathrm{R}_{2}=\mathrm{OCH}_{3} ; \mathrm{R}_{3}=\mathrm{H}$<smiles></smiles>

7<smiles>CC(C)[C@H]1CCC2(C)CC[C@]3(C)C(CCC4C5(C)CC[C@H](O)C(C)(C)C5CC[C@]43C)C12</smiles><smiles>CCC(/C=C/[C@H](C)C1CCC2C1(C)CCC1C3(C)CCC(O)CC3=CCC12C)C(CC)C(C)C</smiles> 
<smiles>CC1=CCC2CC1C2</smiles>

10<smiles>C=C1CC/C=C(\C)CCC2CC1CC2(C)C</smiles>

16<smiles>C=C(C)C1CC=C(C)CC1</smiles>

11<smiles>C=CC(=C)CCC=C(C)C</smiles>

12<smiles>CC1=CCC2C(C)CCC(C(C)C)[C@H]2C1</smiles>

13<smiles>C=C1CCC2OC2CCC2CC1CC2(C)C</smiles>

18<smiles>CCC=C1CCC(=C(C)C)CC=C(C)C1</smiles>

14<smiles>C=C1C=CC(C(C)C)CCC(C)=CCC1</smiles>

15<smiles>COc1ccc2ccc(=O)oc2c1CC=C(C)C</smiles>

20<smiles>C=C1CCC2C(C)CC2C2CC[C@@](C)(O)C12</smiles>

17<smiles>COc1cc2ccc(=O)oc2c2c1OC(C)(C)C=C2</smiles>

21<smiles>CC(C)=CCC/C(C)=C/COc1ccc2ccc(=O)oc2c1</smiles>

19<smiles>COc1c2ccoc2nc2c(OC)cccc12</smiles>

22
O presente estudo avaliou a atividade antimicrobiana de extratos e substâncias obtidas de diferentes partes das espécies de Zanthoxylum stelligerum Turcz. e Spiranthera odoratissima A. St Hil. contra bactérias e fungos.

\section{MATERIAL E MÉTODOS}

\section{Material botânico}

Os espécimes de Spiranthera odoratissima A. St Hil. e Zanthoxylum stelligerum Turcz., Rutaceae, foram coletados no município de Mucugê-BA, Chapada Diamantina. Os exemplares coletados foram identificados pela Profa. Maria Lenise da Silva Guedes e suas exsicatas foram depositadas sob os números 038109 e 040919 , respectivamente, no Herbário Alexandre Leal Costa do Instituto de Biologia da Universidade Federal da Bahia (ALCB).

Os extratos vegetais foram obtidos no Laboratório de Pesquisa em Matéria Médica (LAPEMM) na Faculdade de Farmácia da Universidade Federal da Bahia (UFBA), de acordo com Freitas et al. (2003) e Silva et al. (2002).

\section{Avaliação da atividade microbiana}

Foram utilizados para avaliação da atividade antimicrobiana, os extratos metanólico e hexânico do caule e a substância pura diidroqueleritrina, um alcalóide benzofenantridínico - todos obtidos da espécie $Z$. stelligerum. Da espécie $S$. odoratissima, foram testados os extratos hexânico e metanólico da folha, caule e rizoma da espécie, bem como o extrato clorofórmico da folha.

Os testes microbiológicos foram realizados no Laboratório de Pesquisa em Microbiologia Clínica (LPMC) da Faculdade de Farmácia da UFBA, utilizando os seguintes microrganismos cepas-padrão ATCC (“American Type Culture Collection"): Staphylococcus aureus (ATCC 6835), Micrococcus luteus (ATCC 9341), Escherichia coli (ATCC 10536), Pseudomonas aeruginosa (ATCC 15442), Salmonella cholerea suis (ATCC 10708) e Candida albicans (ATCC 10231) (NCCLS, 2002).

Caso apresentassem resultados positivos de inibição, os extratos também seriam testados frente às amostras clínicas (isolados clínicos): Streptococcus mutans, Streptococcus grupo C, Enterococcus faecalis, Rhodococcus equi, Candida albicans, C. tropicalis e C. guielhermondii.

As soluções-teste foram preparadas a partir dos extratos brutos, dissolvendo-os em solvente apropriado até obter-se uma solução com concentração final de $100 \mu \mathrm{g} /$ $\mu \mathrm{L}$. O solvente utilizado para a dissolução foi o mesmo utilizado para a preparação dos extratos, com exceção dos extratos hexânicos que foram dissolvidos em clorofórmio. 
O método utilizado para avaliação da atividade antimicrobiana foi por difusão em disco de papel de filtro (com diâmetros de $6 \mathrm{~mm}$ ) impregnados com alíquotas de $10 \mu \mathrm{L}$ da solução-teste de cada extrato dissolvido. Foram adicionados $10 \mu \mathrm{L}$ para a obtenção de $1000 \mu \mathrm{g}$ finais no disco e 20 e $30 \mu \mathrm{L}$, para obtenção de 2000 e $3000 \mu \mathrm{g}$, respectivamente. Entre as aplicações das alíquotas de 10 $\mu \mathrm{L}$, os discos foram secos em estufa a $35^{\circ} \mathrm{C}$ por 5 minutos (Bauer, 1966; Navarro \& Delgado, 1999; Ngane et al., 2000).

As suspensões microbianas foram preparadas, colocando-se cinco unidades formadoras de colônias (UFC) em $5 \mathrm{~mL}$ de solução salina esterilizada $(0,9 \%$ de $\mathrm{NaCl}$ ) comparadas e ajustadas à escala padrão 0,5 de McFarland. Em seguida, com auxílio de um swab esterilizado, foram semeadas em superfície de uma placa de Petri contendo ágar Mueller-Hinton, para as bactérias e ágar Sabouraud, para as leveduras. Após as semeaduras, os discos foram então aplicados com auxílio de uma pinça e as placas foram incubadas em condições de aerobiose a $37{ }^{\circ} \mathrm{C}$, por $24 \mathrm{~h}$ para as bactérias, com exceção do $S$. mutans e Streptococcus Grupo C, que foram incubadas em condições de microaerofilia (5 a $10 \%$ de $\mathrm{CO}_{2}$ ). As placas semeadas com as leveduras foram incubadas a temperatura de $28^{\circ} \mathrm{C}$, por $48 \mathrm{~h}$ (Barry et al., 1996; Bauer et al., 1966; Navarro \& Delgado, 1999; Ngane et al., 2000).

Após o período de incubação, as zonas de inibição de crescimento microbiano foram medidas e registradas em milímetros de diâmetro. Foram utilizados controles positivos (discos com $100 \mu \mathrm{g}$ de tetraciclina e gentamicina, para as bactérias e com $1000 \mu \mathrm{g}$ de cetoconazol, para as leveduras) e controles negativos (discos com os solventes orgânicos usados nas soluções dos extratos).

\section{RESULTADOS}

Nas Tabelas 1 e 2, são apresentados os resultados da atividade antimicrobiana de Zanthoxylum stelligerum para microrganismos padronizados e isolados clínicos. Esta espécie apresentou uma discreta atividade frente $S$. aureus, cerca de 7 e $9 \mathrm{~mm}$ para os extratos metanólico e clorofórmico do caule, respectivamente, se comparada ao controle positivo ( $30 \mathrm{~mm}$ de halo).

O extrato metanólico da raiz demonstrou atividade antibacteriana frente ao S. aureus e M. luteus (14 e $19 \mathrm{~mm}$, respectivamente), bem como atividade antifúngica frente às leveduras do gênero Candida (Tabela 2).

Tabela 1. Atividade antimicrobiana in vitro de Zanthoxylum stelligerum Turcz. em milímetros de diâmetro.

\begin{tabular}{|c|c|c|c|c|c|c|c|c|c|c|}
\hline \multirow{3}{*}{ Cepas } & \multicolumn{5}{|c|}{ Diidroqueleritrina } & \multicolumn{5}{|c|}{ Extrato metanólico da raiz } \\
\hline & \multicolumn{3}{|c|}{$\mathrm{x} 10^{3} \mu \mathrm{g} /$ disco } & \multicolumn{2}{|c|}{ Controle } & \multicolumn{3}{|c|}{$\mathrm{x} 10^{3} \mu \mathrm{g} /$ disco } & \multicolumn{2}{|c|}{ Controle } \\
\hline & 1 & 2 & 3 & $\mathrm{C}$ & $\mathrm{C}$ & 1 & 2 & 3 & $\mathrm{C}$ & $\mathrm{C}$ \\
\hline Staphylococcus aureus ${ }^{1}$ & 16 & 17 & 17 & 30 & - & 10 & 12 & 14 & 31 & - \\
\hline Micrococcus luteus ${ }^{1}$ & 21 & 21 & 19 & 3 & - & 12 & 15 & 19 & 35 & - \\
\hline Streptococcus mutans ${ }^{2}$ & 10 & 12 & 12 & 37 & - & - & 7 & 8 & 36 & - \\
\hline Enterococcus faecalis ${ }^{2}$ & 13 & 14 & 14 & 25 & - & 8 & 9 & 11 & 25 & - \\
\hline Streptococcus Grupo C ${ }^{2}$ & 9 & 9 & 10 & 8 & - & - & - & - & - & - \\
\hline Rhodococcus equi ${ }^{2}$ & 7 & 9 & 9 & 8 & - & - & - & - & 7 & - \\
\hline Escherichia coli ${ }^{1}$ & 13 & 13 & 16 & 33 & - & - & - & - & 32 & - \\
\hline Pseudomonas aeruginosa $^{1}$ & - & - & - & 29 & - & - & - & - & 30 & - \\
\hline Salmonella cholerea suis ${ }^{1}$ & - & - & - & 30 & - & - & - & - & 31 & - \\
\hline
\end{tabular}

$\mathrm{C}^{+}$: Tetraciclina e Gentamicina (100 $\left.\mu \mathrm{g}\right)$; C- : solvente da solução teste (100 $\mu \mathrm{L} /$ disco); (-) nenhum halo observado; 1 . Amostras ATCC; 2. Isolados de material clínico

Tabela 2. Atividade antimicrobiana in vitro do extrato metanólico da raiz de Zanthoxylum stelligerum Turcz. em milímetros de diâmetro.

\begin{tabular}{lccccc}
\hline & \multicolumn{3}{c}{} & \multicolumn{2}{c}{ Controle } \\
\cline { 2 - 6 } Cepas & 1 & 2 & 3 & $\mathrm{C}$ & $\mathrm{C}$ \\
\hline${\text { Candida } \text { albicans }^{1}}$ & 9 & 12 & 13 & 18 & - \\
${\text { Candida } \text { albicans }^{2}}^{2}$ & 8 & 9 & 11 & 20 & - \\
Candida tropicalis $^{2}$ & 12 & 14 & 16 & 17 & - \\
Candida guielhermondii $^{2}$ & 8 & 10 & 12 & 18 & - \\
\hline
\end{tabular}

$\mathrm{C}^{+}$: Cetoconazol $(1000 \mu \mathrm{g}) ; \mathrm{C}:$ solvente da solução teste $(100 \mu \mathrm{L} /$ disco); (-) nenhum halo observado; 1 . Amostras ATCC; 2. Isolados de material clínico. 
$\mathrm{O}$ alcaloide diidroqueleritrina exibiu um espectro de ação mais amplo que o perfil do extrato que o originou, o extrato metanólico da raiz. Observou-se também atividade antibiótica frente a bactérias Gram positivas e seus isolados clínicos, além de apresentar inibição ante a cepa-padrão $E$. coli, uma bactéria Gram negativa. Por não apresentarem resultados de inibição para bactérias Gram negativas, os extratos foram testados somente para isolados clínicos de bactéria Gram positivas. Nenhum dos sete extratos de $S$. odoratissima apresentou atividade antimicrobiana frente aos microrganismos testados.

\section{DISCUSSÃO}

A inibição do crescimento de microrganismos in vitro observada no extrato metanólico da raiz e no alcaloide purificado de Zanthoxylum stelligerum Turcz. indica ser a diidroqueleritrina, uma das substâncias ativas responsáveis por esta atividade.

Inúmeros estudos de atividade antimicrobiana de espécies do gênero Zanthoxylum têm apresentado atividade bactericida, a exemplo de $Z$. tetraspermun (Nissanka et al., 2001), Z. clava-herculi (Gibbons et al., 2003) e Z. rhoifolium (Gonzaga et al., 2003), nas quais foram encontrados alcaloides benzofenantrídinicos de estruturas similares à diidroqueleritrina. Outro alcaloide benzofenantridínico, a sanguinarina, encontrado nas raízes de Sanguinaria canadensis Linné, Papaveraceae, apresenta a capacidade de inibir a aderência de bactérias na formação da placa dental e é utilizado em dentifrícios e enxaguatórios bucais na prevenção de periodontite (Godowski, 1989; Robbers et al., 1997).

Diante das evidências, a atividade antimicrobiana da diidroqueleritrina é considerada um resultado esperado. Este alcaloide representa um grande potencial como candidato a um novo fármaco ou protótipo e será submetido a outros ensaios biológicos frente aos fungos filamentosos e às novas cepas bacterianas isoladas de amostras clínicas.

A espécie Spiranthera odoratissima A. St Hil. é utilizada na medicina tradicional para o tratamento de infecções bacterianas, a exemplo de sífilis, furúnculo e acne (Terezan et al., 2007) e apresenta atividade antiinflamatória comprovada (Matos et al., 2003). Por esta razão, a potencialidade de $S$. odoratissima como fonte de novos fármacos não pode ser descartada, apesar desta não apresentar atividade de inibição perante aos microrganismos testados.

\section{AGRADECIMENTOS}

Os autores agradecem à FAPESB, CNPq e Banco do Nordeste pelas bolsas e apoios financeiros concedidos.

\section{REFERÊNCIAS}

Alves HM 2001. A diversidade química das plantas como fonte de fitofármacos. Cad Temat QNEsc 3: 10-15.

Barry AL, Brow SD 1996. Fluconazole disk diffusion procedure for determining susceptibility of Candida species. J Clin Microbiol 34: 2154-2157.

Bauer AW, Kirby WMM, Sherris JC, Turck M 1966. Antibiotic sensitivity testing a standardized single disk method. $\mathrm{Am}$ J Clin Pathol 45: 493- 496.

Benghezal M, Adam E, Lucas A, Burn C, Orchard MG, Deuschel C, Valentino E, Braillard S, Paccaud JP, Cosson P 2007. Inhibitors of bacterial virulence identified in a surrogate host model. Cell Microbiol 9: 1336-1342.

Dewick PM 2002. Medicinal natural products: a biosynthetic approach. London: John Wiley \& Sons.

Freitas CMJ, Lucchese AM, Silva FS, Velozo ES 2003. Coumarins, furoquinoline alkaloids and terpenes from Spiranthera odoratissima (Rutaceae). Biochem Syst Ecol 31: 805-807.

Gibbons S, Leimkugel J, Oluwatuyi M, Heinrich M 2003. Activity of Zanthoxylum clava-herculis extracts against multi-drug resistant methicillin-resistant Staphylococcus aureus (mdr-MRSA). Phytother Res 17: 274-275.

Gibbs, RD 1974. Chemotaxonomy of Flowering Plants. Montreal/ London: McGill-Queen's University Press.

Godowski KC 1989. Antimicrobial activity of sanguinarine. $J$ Clin Dent 1: 96-101.

Gonzaga WA, Weber AD, Giacomelli SR, Dalcol II, Hoelzel SC, Mordel AF 2003. Antibacterial alkaloids from Zanthoxylum rhoifolium. Planta Med 69: 372-374.

Haida KS, Parzianello L, Werner S, Garcia DR, Inácio CV 2007. Avaliação in vitro da atividade antimicrobiana de oito espécies de plantas medicinais. Arq Cienc Saude Unipar 11: 185-192.

Hoefel R, Vidotti CCF, Menezes ES, Pinheiro S 2006. Ações que estimulam o uso racional de antimicrobianos. Bol Farmacot 11: 1-4.

Matos LG, Pontes IS, Tresvenzol LM, Paula JR, Costa EA 2003. Atividades analgésica e/ou antiinflamatória da fração aquosa do extrato etanólico das folhas de Spiranthera odoratissima A. St. Hillaire (Manacá). Rev Bras Farmacogn 13: 15-16.

Moreira LB 2004. Princípios para uso racional de antimicrobianos. Rev AMRIGS 48: 118-120.

NCCLS 2002. Performance Standards for Antimicrobial Disk Susceptibility Tests and Reference method for broth dilution antifungal susceptibility testing of yeasts (approved Standard M2-A4). Villanova, PA, National Committee for Clinical Laboratory Standards.

Navarro V, Delgado G 1999. Two antimicrobial alkaloids from Bocconia arborea. J Ethnopharmacol 66: 223-226.

Ngane AN, Biyiti L, Zollo PH, Bouchet P 2000. Evaluation of antifungal activity of extracts of two Cameroonian Rutaceae: Zanthoxylum leprieurii Guikk. et Perr. and Zanthoxylum xanthoxyloides Waterm. J Ethnopharmacol 70: 335-342.

Nissanka AP, Karunaratne V, Bandara BM, Kumar V, Nakanishi T, Nishi M, Inada A, Tillekeratn, LM, Wijesundar 
DS, Gunatilaka AA 2001. Antimicrobial alkaloids from Zanthoxylum tetraspermum and Z. caudatum. Phytochemistry 56: 857-861.

Oliveira AC, Silva RS 2008. Desafios de cuidar em saúde frente à resistência bacteriana: uma revisão. Rev Elet Enferm 10: 189-197.

Oliveira EL, Freitas PC, Guedes ML, Velozo ES 2002. Estudo fitoquímico de Zanthoxylum stelligerum (Turcz). Rev Bras Farmacogn 12: 29-30.

Robbers JE, Speedie MK, Tyler VE 1997. Farmacognosia \& Farmacobiotecnologia. São Paulo: Editora Premier.

Silva CV, Velozo ES, Guedes ML 2002. Micromoléculas de Zanthoxylum stelligerum (Turcz). Rev Bras Plant Med 5: $75-78$.

Silva CV 2006. Alcalóides benzofenantridinicos e outros metabólitos do caule e frutos de Zanthoxylum tingoassuiba St. Hil. Salvador, 112p. Dissertação de Mestrado - Programa de Pós-graduação em Química, Universidade Federal da Bahia.

Terezan AP, Fernandes JB, Vieira PC, Silva MFGF, Pagnocca FC, Almeida RNA, Bueno OC, Rossi RA 2007. Isolamento das substâncias do extrato bioativo obtido dos galhos de Spiranthera odoratíssima St. Hil. 30 ${ }^{a}$. Reunião Anual da $S B Q$. Águas de Lindóia, Brasil.

Wang J, Soisson SM, Young K, Shoop W, Kodali S, Galgoci A, Painter R, Parthasarathy G, Tang YS, Cummings R, Ha S, Dorso K, Motyl M, Jayasuriya H, Ondeyka J, Herath K, Zhang C, Hernandez L, Allocco J, Basilio A, Tormo JR, Genilloud O, Vicente F, Pelaez F, Colwell L, Lee SH, Michael B, Felcetto T, Gill C, Silver LL, Hermes JD, Bartizal K, Barrett J, Schmatz D, Becker JW, Cully D, Singh SB 2006. Platensimycin is a selective FabF inhibitor with potent antibiotic properties. Nature 441: 358-361.

Wannmacher L 2004. Uso indiscriminado de antibiótico e resistência microbiana: Uma guerra perdida? URM: Temas Selecionados 1: 1-6. 